\title{
Ayuda oficial española al desarrollo: Los retos de la especialización geográfica y sectorial
}

\author{
JOSÉ MARÍA LARRÚ \\ Departamento de Economía Aplicada, UNIVERSIDAD CEU SAN PABLO, ESPAÑA. E- \\ mail: larram@ceu.es
}

\section{SERGIO TEZANOS VÁZQUEZ}

Departamento de Economía y Cátedra de Cooperación Internacional y con Iberoamérica, UNIVERSIDAD DE CANTABRIA,ESPAÑA.E-mail: tezanoss@unican.es

\begin{abstract}
RESUMEN
España cierra un ciclo de fuerte expansión de su política pública de ayuda internacional para el desarrollo, e inicia una nueva etapa de crisis y ajuste presupuestario en la que deberá revisar su estrategia de cooperación. En este artículo se describen los hechos estilizados que caracterizan la estructura de la Ayuda Oficial española al Desarrollo (AOD). Para ello se analiza la distribución geográfica, sectorial e instrumental de la AOD, comparando los niveles de coordinación logrados por los dos últimos Gobiernos del PP y del PSOE, y se proponen criterios de política económica para diseñar una política pública de cooperación verdaderamente selectiva y “estratégica”.
\end{abstract}

Palabras clave: Ayuda Oficial al Desarrollo (AOD), España, distribución geográfica, distribución sectorial.

\section{Spanish Official Development Assistance: The Geographical and Sector Specialization Challenges}

\begin{abstract}
Spain closes a cycle of strong growth in its public policy of foreign aid for development, and starts a new period of crisis and fiscal adjustment in which the co-operation strategy must be revised. This article describes the stylized facts that characterize the Spanish Official Development Assistance (ODA). We analyze the geographical, sectorial and instrumental ODA distribution, comparing the different patterns followed by the last two Governments of the PP and the PSOE, and we propose economic policy criteria to design a truly selective and "strategic" public policy of international co-operation.
\end{abstract}

Keywords: Official Development Assistance (ODA), Spain, Geographical Distribution, Sectorial Distribution.

Clasificación JEL: F35, O19, I30, C5

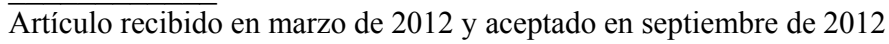

Artículo disponible en versión electrónica en la página www.revista-eea.net, ref. ə-30302 


\section{INTRODUCCIÓN}

Cerrado el ciclo de expansión de los recursos de Ayuda Oficial al Desarrollo (AOD), e inmersa en un proceso de crisis y ajuste presupuestario, España afronta ahora el reto de actualizar su política pública de cooperación internacional para compensar -siquiera parcialmente- la importante reducción de los recursos con aumentos en la calidad y el impacto de las actuaciones. Esta actualización pasa, inevitablemente, por revisar los aspectos "económicos" de la distribución de la AOD, tanto a nivel geográfico (entre países en desarrollo), como sectorial e instrumental (entre sectores e instrumentos), al objeto de definir una "estrategia de especialización" de la cooperación pública española que sea verdaderamente selectiva y eficaz.

No en vano, la eficacia de la AOD española ha estado en tela de juicio prácticamente desde sus inicios. De acuerdo con los índices sobre calidad de la ayuda aparecidos recientemente, España se sitúa en posiciones relativamente bajas: ocupa el décimo puesto (sobre 22 donantes, en orden creciente de calidad) en el Índice Ajustado de Calidad de la Ayuda; entre el 21 y el 26 de 31 donantes en el QuODA; el 24 de 30 en calidad de la ayuda en salud; el 25 de 38 en el índice de calidad de la ayuda del Banco Mundial; el 34 de 42 en el índice de buenas prácticas; el 15 de 19 en Respuesta Humanitaria y el 44 de 58 en el Índice de Transparencia ${ }^{1}$.

Dados estos retos de calidad, en este artículo analizamos la distribución geográfica, sectorial e instrumental de la AOD española, con el objetivo de identificar las causas de sus elevados niveles de dispersión e identificar los países, sectores e instrumentos en los que, de facto, se ha producido una cierta "especialización" española y en los que se debería tender a concentrar los recursos para incrementar el impacto en un contexto de reducción presupuestaria.

El análisis se acota a los recursos públicos de la cooperación internacional española (la denominada AOD) financiada tanto por la Administración General del Estado (AGE), como por las Comunidades Autónomas (CCAA), los Entes Locales (EELL) y las Universidades públicas. En concreto, la AGE aporta la mayor parte de la AOD (más de cuatro quintas partes de los recursos), mientras que el escaso quinto restante lo financian y gestionan las CCAA, los EELL y las universidades ${ }^{2}$. No obstante es importante destacar que esta parcial "descentralización" de la gestión de la AOD no responde a una transferencia de

\footnotetext{
${ }^{1}$ Para un mayor detalle de estos índices y sus fuentes véase el informe sobre la cooperación española de Olivié et al. (2011, págs 30-31). Para el índice de Respuesta Humanitaria véase DARA (2011) y para la calidad de la ayuda en salud, véase Durán y Glassman (2012).

${ }^{2}$ En promedio, para el periodo con información facilitada por los sucesivos Seguimientos del Plan Anual de Cooperación Internacional (1997-2009), el 86\% de los recursos los gestionó la AGE, el 9,5\% las CCAA, el 4,25\% los EELL y el 0,25\% restante las universidades públicas.
} 
competencias de cooperación por parte de la AGE, sino a un intento de aunar mayores recursos y de acercar esta política pública a la ciudadanía que la financia, tal y como recoge la Ley Española de Cooperación Internacional para el Desarrollo (Ley 13/1998 de 7 de julio). Es más, dicha Ley atribuye al Ministerio de Asuntos Exteriores la realización de la política española de cooperación al desarrollo bajo el principio constitucional de unidad de acción en el exterior (arts. 3 y 17), si bien el carácter parcialmente descentralizado de la cooperación española impide un control absoluto del Gobierno de España sobre la planificación y la ejecución de los recursos. En última instancia, el estudio parte del convencimiento de que la política oficial de cooperación es una "política de Estado", cuya coherencia debe evaluarse en relación con el conjunto de la AOD española ${ }^{3}$.

El presente análisis aporta la novedad de considerar la "dimensión política" de la gestión de la AOD, distinguiendo y comparando dos periodos de Gobierno con signos políticos distintos: las dos legislaturas consecutivas del PP (19962004) y las dos del PSOE (2004-2011). Más concretamente, como criterio general analizamos dos periodos políticos bien acotados: 1997-2003 (que incluye las dos legislaturas de Gobierno del PP) y 2005-2009 (las dos legislaturas de Gobierno del PSOE) ${ }^{4}$. Excluimos del análisis los años de celebración de elecciones (1996 y 2004) por tratarse de periodos de transición política en los que ninguno de los dos Gobiernos gestionó autónomamente la AOD. De este modo, el objetivo central de la investigación es contrastar en qué medida cada una de las administraciones políticas (del PP y del PSOE) han logrado coordinar la distribución (geográfica, sectorial e instrumental) de la ayuda española financiada por las distintas administraciones públicas, partiendo del supuesto (reconocido por la Ley de Cooperación) de que la AGE (por medio del Ministerio de

\footnotetext{
${ }^{3}$ El art. 4 de la Ley de Cooperación Internacional para el Desarrollo afirma que, en relación con el principio de coherencia, "los principios y objetivos señalados en los artículos anteriores informarán todas las políticas que apliquen las Administraciones públicas en el marco de sus respectivas competencias...". Y el art. 20.2. sostiene que la Cooperación de las CCAA y EELL debe hacerse "debiendo respetar las líneas generales y directrices básicas establecidas por el Congreso de los Diputados a que se refiere el art. 15.1 [que es el que dictamina que es "al Congreso a quien corresponde establecer cada cuatro años, en la forma y modo que se determine y a propuesta e iniciativa del Gobierno, las líneas generales y directrices básicas de la política española de cooperación internacional para el desarrollo"] y el principio de colaboración entre Administraciones públicas...". Por tanto, aunque parte de las evidencias que aquí se ofrecen están influidas por las decisiones de la cooperación descentralizada y universitaria, nosotros tomamos los datos como representación de la política española dirigida por un Gobierno que elaboró los respectivos Planes Directores según el mandato del art. 15.1. de la Ley 23/1998 de 7 de julio.

${ }^{4}$ En el análisis utilizamos la información suministrada por la base de datos del Comité de Ayuda al Desarrollo (CAD). Dados los dilatados plazos de elaboración de las estadísticas de ayuda, 2009 fue el último año de información disponible durante el periodo de elaboración de este análisis (finales de 2011).
} 
Asuntos Exteriores) es responsable de la dirección y coordinación del conjunto de la AOD española -entre otras razones, para limitar el impacto potencialmente negativo que puede ejercer la estructura descentralizada del Estado español sobre la fragmentación de los recursos.

El artículo se estructura de la siguiente manera: tras este epígrafe introductorio, en la segunda sección analizamos la distribución geográfica de la AOD, tanto en términos de "regiones", como de "niveles de renta" de los países socios. En el tercer epígrafe analizamos la distribución sectorial, para lo que explicamos los rasgos estructurales de dicha asignación, medimos la volatilidad del reparto sectorial e identificamos los 20 sectores con mayor financiación relativa. En el cuarto epígrafe explicamos el cuadro de instrumentos de la AOD española. Finalmente, el artículo concluye resumiendo los principales resultados de la investigación y proponiendo algunas medidas de política económica que podrían dotar de mayor carácter estratégico a la política pública española de cooperación internacional.

\section{DISTRIBUCIÓN GEOGRÁFICA DE LA AOD ESPAÑOLA}

Para analizar la asignación geográfica de la AOD española procedemos en tres pasos. En primer lugar, describimos la asignación de los fondos de ayuda por regiones geográficas; en segundo lugar, analizamos la distribución por niveles de renta per capita de los países receptores; $\mathrm{y}$, en tercer lugar, estudiamos el número total de países receptores y verificamos si esta asignación ha seguido las prioridades de los sucesivos Planes Directores de la Cooperación Española.

\subsection{Distribución de la AOD por regiones}

Por continentes, la ayuda española se dirige mayoritariamente a América, seguida -a gran distancia- de África, Asia y Europa (Tabla 1). Aunque la distribución no ha sufrido grandes cambios entre el periodo de Gobierno del PP y del PSOE, lo más destacado ha sido la reducción de las aportaciones a América $(9,5$ puntos porcentuales menos en el periodo 2005-2009), siendo Centroamérica la subregión que más pierde (-9,3 puntos porcentuales). Esta disminución, a su vez, ha permitido aumentar las participaciones de África $(+6,2)$ y Asia $(+0,8)$.

En términos subregionales, ambas administraciones priorizaron a Sudamérica y a Norte y Centro América. No obstante, durante la administración del PSOE se incrementaron notablemente las cuotas de ayuda de África Subsahariana ( +3.2 puntos porcentuales) y Oriente Medio $(+3.5)$, a costa de reducir las cuotas de Norte y Centro América, Sudamérica y Extremo Oriente. 
Tabla 1

Asignación de la AOD española por regiones geográficas (\%)

\begin{tabular}{|l|c|c|c|}
\hline Región y subregión & $\begin{array}{c}\mathbf{1 9 9 7 - 2 0 0 3} \\
\text { PP }\end{array}$ & $\begin{array}{c}\mathbf{2 0 0 5 - 2 0 0 9} \\
\text { PSOE }\end{array}$ & $\begin{array}{c}\text { Diferencia } \\
\text { (2005-09)-(1997-03) }\end{array}$ \\
\hline EUROPA & $7,2 \%$ & $4,4 \%$ & $-2,7 \%$ \\
\hline AFRICA & $21,4 \%$ & $27,7 \%$ & $6,2 \%$ \\
\hline Norte de África & $5,9 \%$ & $7,0 \%$ & $1,1 \%$ \\
\hline África Subsahariana & $15,4 \%$ & $18,7 \%$ & $3,2 \%$ \\
\hline África, regional & $0,1 \%$ & $2,0 \%$ & $1,9 \%$ \\
\hline AMÉRICA & $45,7 \%$ & $36,2 \%$ & $-9,5 \%$ \\
\hline Norte y Centro América & $25,9 \%$ & $16,6 \%$ & $-9,3 \%$ \\
\hline Sudamérica & $15,0 \%$ & $12,7 \%$ & $-2,3 \%$ \\
\hline América, regional & $4,8 \%$ & $6,9 \%$ & $2,1 \%$ \\
\hline ASIA & $13,8 \%$ & $14,6 \%$ & $0,8 \%$ \\
\hline Extremo Oriente & $8,2 \%$ & $3,4 \%$ & $-4,8 \%$ \\
\hline Asia del Sur y Central & $1,6 \%$ & $3,2 \%$ & $1,7 \%$ \\
\hline Oriente Medio & $3,9 \%$ & $7,4 \%$ & $3,5 \%$ \\
\hline Asia, regional & $0,2 \%$ & $0,6 \%$ & $0,4 \%$ \\
\hline OCEANíA & $0,0 \%$ & $0,0 \%$ & $0,0 \%$ \\
\hline SIN ESPECIFICAR & $11,9 \%$ & $17,1 \%$ & $5,2 \%$ \\
\hline
\end{tabular}

Fuente: Elaboración propia con datos del CAD (2011b). Cálculos con desembolsos de AOD neta en dólares constantes de 2009.

Este cambio en la asignación de la AOD durante el Gobierno socialista respondió, en buena medida, a la definición de una nueva estrategia española de política exterior en el continente africano, que se plasmó en la aprobación de dos "Planes África" de aplicación en los periodos 2006-2008 y 2009-2012. Estos planes trataron de proyectar, de manera global, la política exterior española hacia la región -no únicamente la cooperación para el desarrollo-. No obstante, los países africanos priorizados por estos planes terminaron sumándose a las prioridades identificadas por la cooperación oficial española, lo que ha resultado en una "suavización" de la especialización histórica española en América Latina, y la incorporación de nuevos socios africanos con ingresos más bajos (en última instancia, los priorizados por la agenda de los Objetivos de Desarrollo del Milenio).

\subsection{Distribución de la AOD por niveles de renta}

Los países de renta media-baja han sido los principales destinatarios de la AOD española en ambos periodos (Tabla 2). Sin embargo, durante el periodo de Gobierno socialista (2005-09) se ha producido un incremento de 4,7 puntos porcentuales en la ayuda destinada a los países menos adelantados y un descenso de $-4,4$ puntos en los países de renta media-alta. Esta redistribución hacia 
los países más pobres resulta más coherente tanto con el "objetivo central" que recoge la Ley Cooperación Internacional de contribuir a la "erradicación de la pobreza en el mundo en todas su manifestaciones" (artículo 1), como con los Objetivos de Desarrollo del Milenio. ${ }^{5}$

Tabla 2

Asignación de la AOD española por niveles de renta de los receptores

\begin{tabular}{|l|c|c|c|}
\hline \multicolumn{1}{|c|}{ Zona geográfica } & $\begin{array}{c}\mathbf{1 9 9 7 - 2 0 0 3} \\
\text { PP }\end{array}$ & $\begin{array}{c}\mathbf{2 0 0 5 - 2 0 0 9} \\
\text { PSOE }\end{array}$ & $\begin{array}{c}\text { Diferencia } \\
\text { (2005-09)-(1997-03) }\end{array}$ \\
\hline Países menos adelantados & $11,3 \%$ & $16,0 \%$ & $4,7 \%$ \\
\hline Países de renta baja & $4,8 \%$ & $4,3 \%$ & $-0,5 \%$ \\
\hline Países de renta media-baja & $54,2 \%$ & $46,9 \%$ & $-7,3 \%$ \\
\hline Países de renta media-alta & $11,7 \%$ & $7,3 \%$ & $-4,4 \%$ \\
\hline Sin especificar & $18,05 \%$ & $25,61 \%$ & $4,6 \%$ \\
\hline
\end{tabular}

Fuente: Elaboración propia con datos del CAD (2011b). Cálculos con desembolsos de AOD neta en dólares constantes de 2009.

Una implicación fundamental de la priorización histórica de España por los países de rentas medias es la existencia de un "sesgo regresivo" en la asignación de la AOD española, lo que contrasta con los patrones distributivos del agregado de países donantes de la $\mathrm{UE}$ y del $\mathrm{CAD}$, que distribuyen mas progresivamente sus ayudas (Tezanos, 2008a). No obstante, la regresividad del reparto de la AOD se ve parcialmente modulada al evaluarse el subgrupo de países que comparten vínculos históricos con España, con los cuales, según los informes de evaluación entre pares del CAD (2002, 2007 y 2012), la cooperación española presenta cierta "ventaja comparativa". De este modo, la distribución de la AOD de España ha sido especialmente progresiva con este subgrupo de países. En última instancia, debe entenderse que la especial atención que concede España a los países con los que comparte vínculos históricos (mayoritariamente países de renta media con tamaños poblacionales comparativamente reducidos) determina, en realidad, tanto la "especialización" en países de renta media, como el sesgo a favor de los países pequeños (Tezanos, 2008a y 2008b, y Tezanos y Gutierrez, 2011).

\footnotetext{
${ }^{5}$ Este análisis simplifica la evaluación de la progresividad de la ayuda utilizando únicamente el indicador de renta per capita que facilita el CAD. Véase Torres y Allepuz (2009) para un análisis más matizado de otros indicadores de desarrollo humano que podrían utilizarse para realizar un análisis más preciso de la distribución geográfica de la AOD en función de los niveles de vida de los receptores.
} 


\subsection{Asignación planificada vs. asignación realizada}

La lista de países priorizados por los sucesivos Planes Directores de la Cooperación Española (MAE, 2001 y 2005; MAEC, 2009) ha sido siempre extensa. Desde los 32 países del primer Plan Director 2001-2004, se pasó a los 52 del Plan Director 2005-2009, y a los 50 del Plan Director 2009-2012. ${ }^{6}$

Analizando el volumen de AOD recibido por cada país socio, observamos los siguientes hechos estilizados:

En primer lugar, el número de países que efectivamente recibe ayuda ronda el centenar (94 en 1997, que es el mínimo, y 127 en 2009, que es el máximo), lo que duplica el número de países planificados en los respectivos Planes Directores (Figura 1). Por periodos políticos, durante el Gobierno del PSOE (20052009) España cooperó, en promedio, con 116 países socios por año, frente a los 101 del periodo del PP (1997-2003). Este significativo aumento en el número de socios se vio coadyuvado por el incremento absoluto en el presupuesto de AOD de la etapa socialista, que permitió aumentar el número de receptores sin necesidad de reducir los desembolsos de ayuda (en términos absolutos) a los socios históricos de la cooperación española ${ }^{7}$.

En segundo lugar, la Tabla 3 muestra que el promedio de ayudas en el periodo 1997-2003 fue de 15,98 millones de dólares (constantes de 2009) con una volatilidad (medida a través del coeficiente de variación) superior a 1,6 (si excluimos el dato de 2001 que es un año atípico por la condonación de deuda a Nicaragua fruto de una operación triangular con Guatemala ligada al caso Cegulsa $^{8}$ ). En contraste, y confirmando el crecimiento del volumen de AOD, el periodo del PSOE presenta un promedio de 24,71 millones de dólares y un coeficiente de variación de 1,75, destacando la volatilidad de los años 2005-06 en

\footnotetext{
${ }^{6}$ En realidad, el segundo Plan Director priorizó a un total de 73 países, teniendo en cuenta que incluyó dos grupos de países sin especificar en concreto las naciones que los integraban: se trataba de los 13 "países del Sureste Asiático que padecieron las consecuencias del maremoto de 2004" (Indonesia, Malasia, Sri Lanka, Tailandia, Bangladesh, India, Myanmar, Maldivas, Seychelles, Madagascar, Somalia, Tanzania y Kenia) y los ocho "países de renta media-baja candidatos a la adhesión a la UE y los países que pudieran precisar ayuda puntual en el marco del Pacto de Estabilidad para el Sudeste de Europa" (Turquía, Serbia, Montenegro, Croacia, Macedonia, Moldavia, Ucrania y Bielorrusia). Estos agregados permitieron justificar a priori eventuales actuaciones en estas regiones, si bien fueron excluidas del ciclo de planificación.

${ }^{7}$ El efecto no se debe a la cooperación descentralizada, pues, a modo de ejemplo y tomando la ayuda bilateral del último año de cada gobierno -donde el esfuerzo en concentración podría haber sido resultado de mayor voluntad política-, en 2003 la AGE no cooperó en 7 países donde sí lo hizo la descentralizada (100 países la AGE, 71 las CCAA y 68 las EELL). En 2009, la AGE no cooperó en 3 donde sí lo hizo la descentralizada (113 países la AGE, 91 las CCAA y 88 las EELL).

${ }^{8}$ Para detalles sobre este caso véase Larrú (2011) y las referencias allí citadas. Si se incluye el dato de 2001 el CV del periodo es de 1,95.
} 
los que el coeficiente superó el valor de 2 puntos. No obstante -cabe mencionarson las variaciones de los grandes receptores (y no la de los pequeños) las que aportan volatilidad a la AOD española. ${ }^{9}$

Figura 1

Evolución del número de países socios de AOD española

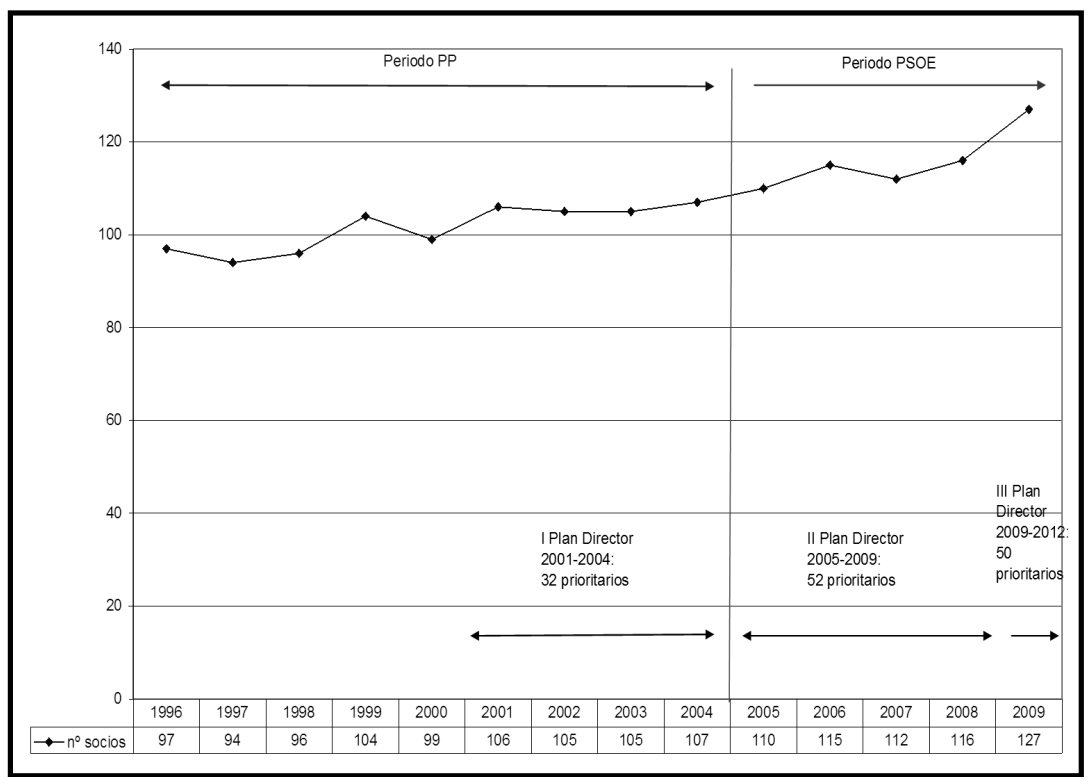

Fuente: Elaboración propia con datos del CAD (2011b).

Tabla 3

Estadísticos descriptivos y principales receptores anuales de la AOD española

\begin{tabular}{|l|c|c|c|c|c|c|c|}
\cline { 2 - 8 } \multicolumn{1}{c|}{} & $\mathbf{1 9 9 7}$ & $\mathbf{1 9 9 8}$ & $\mathbf{1 9 9 9}$ & $\mathbf{2 0 0 0}$ & $\mathbf{2 0 0 1}$ & $\mathbf{2 0 0 2}$ & $\mathbf{2 0 0 3}$ \\
\hline Promedio & 14,23 & 15,25 & 13,04 & 14,96 & 20,83 & 17,13 & 16,39 \\
\hline Des. típica & 24,51 & 25,20 & 20,54 & 26,83 & 80,32 & 25,38 & 25,35 \\
\hline Coef. variación & 1,72 & 1,65 & 1,57 & 1,79 & 3,86 & 1,48 & 1,55 \\
\hline Máximo & 144,27 & 130,34 & 86,00 & 135,97 & 811,44 & 103,19 & 149,74 \\
\hline País máximo & Madagascar & Marruecos & Honduras & Indonesia & Nicaragua & El Salvador & Bolivia \\
\hline
\end{tabular}

${ }^{9}$ Cabe destacar también de la Tabla 3 que los países centroamericanos han sido los que han recibido el mayor volumen de AOD, con la excepción llamativa de los años 1997 (Madagascar) y 2000 (Indonesia), que no fueron considerados países prioritarios. 
Tabla 3 (continuación)

Estadísticos descriptivos y principales receptores anuales de la AOD española

\begin{tabular}{|l|c|c|c|c|c|}
\cline { 2 - 6 } \multicolumn{1}{c|}{} & $\mathbf{2 0 0 5}$ & $\mathbf{2 0 0 6}$ & $\mathbf{2 0 0 7}$ & $\mathbf{2 0 0 8}$ & $\mathbf{2 0 0 9}$ \\
\hline Promedio & 22,72 & 20,19 & 23,38 & 29,36 & 27,91 \\
\hline Des. típica & 47,70 & 41,51 & 37,41 & 45,53 & 40,67 \\
\hline Coef. variación & 2,10 & 2,06 & 1,60 & 1,55 & 1,46 \\
\hline Máximo & 279,77 & 265,08 & 264,98 & 251,16 & 220,04 \\
\hline País máximo & Honduras & Guatemala & Guatemala & Honduras & Marruecos \\
\hline
\end{tabular}

Fuente: Elaboración propia con datos del CAD (2011b). Desembolsos brutos de AOD en dólares constantes de 2009.

En tercer lugar, analizando el conjunto de los 10 países que más suma de AOD (el stock) recibieron durante los periodos del PP y del PSOE se observan tres hechos destacados (Tabla 4):

a) La concentración de AOD en las regiones de Centroamérica y América Andina, seguida por Marruecos y China, en ambos periodos de gestión política.

b) Los casos atípicos que generan las operaciones de alivio de deuda externa: Nicaragua en 2001 (periodo del PP) e Irak en 2005 y 2006 (PSOE).

c) En términos de la concentración de los recursos en los 10 principales receptores, éstos acumularon el $40 \%$ de la ayuda desembolsada en época del PSOE, frente al 45\% del PP (motivado por el caso de Nicaragua ya comentado $^{10}$ ). Estas bajas proporciones acumuladas por los 10 principales receptores son el efecto indirecto de la enorme fragmentación de la AOD repartida en muy pequeñas aportaciones entre un elevado número de socios ${ }^{11}$.

${ }^{10} \mathrm{Su}$ acumulación supone la máxima proporción recibida por un receptor que superó el $10 \%$ de la AOD distribuida a lo largo de todo el periodo.

${ }^{11}$ Según los datos elaborados a partir de los Seguimientos PACI, en 2003 -último ejercicio completo del Gobierno del PP- la AGE financió 2.174 intervenciones (proyectos) que suponen el $44 \%$ del total de las intervenciones bilaterales (excluida la multi-bilateral). Las CCAA financiaron 1.731 proyectos (35\%) y las EELL 988, el 20\% de las 4.893 intervenciones. Por parte del Gobierno del PSOE, en 2009, la AGE financió 6.122 intervenciones bilaterales $(41 \%$ del total), las CCAA 4.416 proyectos (30\%) y las EELL 4.220 proyectos (29\%). La gran diferencia relativa recae en las EELL aunque es probable que esos 9 puntos porcentuales superiores en 2009 respecto a 2003 queden parcialmente explicados por mejoras en el sistema de recolección de la información. Queda pendiente esclarecer las implicaciones de esta proliferación de intervenciones en términos de los costes de transacción (seguimiento, control de intervención y administración) derivados de un aumento de casi 10.000 intervenciones en 6 años. 
Tabla 4

Top-10 de los países receptores de AOD española

\begin{tabular}{|c|c|c|c|c|}
\hline $\begin{array}{c}\text { 1997-2003 } \\
\text { PP }\end{array}$ & País & Stock AOD & $\begin{array}{c}\% \\
\text { AOD geográficamente } \\
\text { distribuida }\end{array}$ & $\begin{array}{c}\% \\
\text { Acumulado }\end{array}$ \\
\hline 1 & Nicaragua & $1.155,34$ & $10,2 \%$ & $10,2 \%$ \\
\hline 2 & Marruecos & 562,01 & $4,9 \%$ & $15,1 \%$ \\
\hline 3 & China & 549,10 & $4,8 \%$ & $19,9 \%$ \\
\hline 4 & Honduras & 511,26 & $4,5 \%$ & $24,4 \%$ \\
\hline 5 & Bolivia & 502,98 & $4,4 \%$ & $28,9 \%$ \\
\hline 6 & Perú & 414,53 & $3,6 \%$ & $32,5 \%$ \\
\hline 7 & El Salvador & 368,68 & $3,2 \%$ & $35,7 \%$ \\
\hline 8 & Ecuador & 364,57 & $3,2 \%$ & $39,0 \%$ \\
\hline 9 & Indonesia & 359,00 & $3,2 \%$ & $42,1 \%$ \\
\hline 10 & Colombia & 330,83 & $2,9 \%$ & $45,0 \%$ \\
\hline
\end{tabular}

\begin{tabular}{|c|c|c|c|c|}
\hline $\begin{array}{c}\text { 2005-2009 } \\
\text { PSOE }\end{array}$ & País & Stock AOD & $\begin{array}{c}\% \\
\text { AOD geográficamente } \\
\text { distribuida }\end{array}$ & $\begin{array}{c}\% \\
\text { Acumulado }\end{array}$ \\
\hline 1 & Guatemala & 940,38 & $6,5 \%$ & $6,5 \%$ \\
\hline 2 & Honduras & 762,72 & $5,3 \%$ & $11,8 \%$ \\
\hline 3 & Marruecos & 641,31 & $4,5 \%$ & $16,3 \%$ \\
\hline 4 & Irak & 627,7 & $4,4 \%$ & $20,7 \%$ \\
\hline 5 & Nicaragua & 608,59 & $4,2 \%$ & $24,9 \%$ \\
\hline 6 & Perú & 562,37 & $3,9 \%$ & $28,8 \%$ \\
\hline 7 & Colombia & 463,55 & $3,2 \%$ & $32,0 \%$ \\
\hline 8 & Bolivia & 420,29 & $2,9 \%$ & $34,9 \%$ \\
\hline 9 & Turquía & 411,67 & $2,9 \%$ & $37,8 \%$ \\
\hline 10 & China & 409,82 & $2,8 \%$ & $40,6 \%$ \\
\hline
\end{tabular}

Fuente: Elaboración propia con datos del CAD (2011b). Desembolsos brutos de AOD en dólares constantes de 2009.

De estos datos puede deducirse que ha habido una concentración de facto en torno a 10 o 12 países, a pesar de que los dos últimos Planes Directores identificaron -cada uno- más de 50. A la vista de esta peculiar distribución de la ayuda española, parece necesaria una lista corta de países verdaderamente prioritarios ya que con una docena de países socios se estaría gestionando (ceteris paribus y siguiendo la tendencia de la última década) más del $40 \%$ de la AOD. En 75 países, de entre los 127 a los que se concedió ayuda entre 2005 y 2009, la cantidad española representó menos del 1\% de la AOD recibida y sólo en 15 supuso más del $20 \%$.

En conclusión, la asignación geográfica de la AOD española se ha caracterizado por su inclinación hacia América (como región históricamente vinculada a España) y África (como región priorizada por la agenda de los Objetivos de 
Desarrollo del Milenio), con cierta tendencia a la equiparación entre ellas durante la etapa del PSOE. En términos de rentas de los receptores, los vínculos históricos de España explican la especial atención concedida a los países de renta media (especialmente los de renta media-baja), que siguen siendo los principales receptores, si bien se incrementaron las ayudas a los países menos adelantados durante el Gobierno socialista. El número total de receptores no ha cesado de crecer, generando mayor fragmentación en los flujos de AOD. La falta de previsibilidad y la excesiva fragmentación elevan los costes de transacción de la AOD, dificultan al receptor una programación eficiente de su desarrollo y distorsionan las decisiones de inversión de los agentes que operan en ese país, todo lo cual puede estar reduciendo la eficacia de estas políticas (Djankov et al., 2009; Tezanos et al., 2009). Por eso, es necesaria una concentración de los flujos de AOD en torno a un número reducido de países realmente prioritarios, resultado de una combinación transparente de criterios tanto políticos, como técnicos (eficiencia) y de desarrollo.

\section{DISTRIBUCIÓN SECTORIAL DE LA AOD ESPAÑOLA}

En esta sección se describen primero los rasgos de la asignación sectorial desagregada en tres dígitos según la clasificación del CAD; a continuación se realiza un análisis de la volatilidad sectorial entre 2002 y 2009; y, finalmente, se comparan los 20 sectores que han recibido más AOD en los periodos de gestión del PP y del PSOE. Conviene alertar que la base de datos del CAD no ofrece información sectorial detallada de los desembolsos anteriores a 2002, lo que exige acortar el periodo de gestión del PP a los años 2002-2003. De todas formas -como se mostrará más adelante-, la asignación sectorial muestra muy poca variación interanual, lo que suaviza el sesgo introducido por esta variación en el primer periodo de estudio.

\subsection{Rasgos estructurales de la asignación sectorial}

La distribución sectorial de la ayuda española en dos periodos regidos por diferentes partidos políticos se ofrece en la Tabla 5.

El principal sector de destino es "infraestructura y servicios sociales", que ganó peso durante la gestión socialista (6,3 puntos porcentuales más). Los dos sectores que experimentan mayores diferencias entre ambos periodos políticos son el de "agua y saneamiento" (que, gracias a la constitución del Fondo para Agua, llegó a representar el 11,1\% en 2009, siendo el sector con mayor porcentaje de todos) y "gobierno y sociedad civil" (que recibió 2,8 puntos porcentuales más en el periodo 2005-09). 
Tabla 5

Distribución sectorial de la AOD española

\begin{tabular}{|c|c|c|c|c|}
\hline \multicolumn{2}{|c|}{ SECTORES CAD } & $\begin{array}{l}\text { 2002-2003 } \\
\text { PP }\end{array}$ & $\begin{array}{l}\text { 2005-2009 } \\
\text { PSOE }\end{array}$ & $\begin{array}{c}\text { Diferencia } \\
(2005-09)-(2002-03)\end{array}$ \\
\hline \multicolumn{2}{|c|}{ 100: I. INFRASTRUCTURA Y SERVICIOS SOCIALES } & $34,3 \%$ & $40,6 \%$ & $6,3 \%$ \\
\hline \multirow{6}{*}{$\begin{array}{l}\text { 100: I. INFRASTRUCTURA } \\
\text { Y SERVICIOS SOCIALES }\end{array}$} & 110: I.1. Educación & $10,5 \%$ & $9,3 \%$ & $-1,1 \%$ \\
\hline & 120: I.2. Salud & $5,3 \%$ & $5,1 \%$ & $-0,2 \%$ \\
\hline & $\begin{array}{l}\text { 130: I.3. Población y salud } \\
\text { reproductiva }\end{array}$ & $0,9 \%$ & $1,9 \%$ & $1,0 \%$ \\
\hline & 140: I.4. Agua y saneamiento & $2,9 \%$ & $7,2 \%$ & $4,3 \%$ \\
\hline & $\begin{array}{l}\text { 150: I.5. Gobierno y Sociedad } \\
\text { Civil }\end{array}$ & $7,2 \%$ & $10,0 \%$ & $2,8 \%$ \\
\hline & $\begin{array}{l}\text { 160: I.6. Otra infraestructura y } \\
\text { servicios sociales }\end{array}$ & $7,5 \%$ & $7,1 \%$ & $-0,4 \%$ \\
\hline \multicolumn{2}{|c|}{ 200: II. INFRASTRUCTURA Y SERVICIOS ECONÓMICOS } & $6,4 \%$ & $10,8 \%$ & $4,4 \%$ \\
\hline \multicolumn{2}{|c|}{ 300: III. SECTORES PRODUCTIVOS } & $6,2 \%$ & $5,2 \%$ & $-1,0 \%$ \\
\hline \multicolumn{2}{|l|}{ 400: IV. MULTISECTORIAL } & $5,4 \%$ & $7,5 \%$ & $2,0 \%$ \\
\hline \multirow{2}{*}{ 400: IV. MULTISECTORIAL } & 410 Medioambiente & $1,5 \%$ & $3,1 \%$ & $1,6 \%$ \\
\hline & 430 Otros multisectorial & $3,9 \%$ & $4,4 \%$ & $0,5 \%$ \\
\hline \multicolumn{2}{|c|}{$\begin{array}{l}\text { 500: VI. AYUDA EN FORMA DE SUMINISTRO DE BIENES Y } \\
\text { AYUDA GENERAL PARA PROGRAMAS }\end{array}$} & $1,9 \%$ & $3,3 \%$ & $1,4 \%$ \\
\hline \multicolumn{2}{|c|}{ 600: VII. ACTIVIDADES RELACIONADAS CON LA DEUDA } & $9,2 \%$ & $13,7 \%$ & $4,5 \%$ \\
\hline \multicolumn{2}{|c|}{ 700: VIII. AYUDA HUMANITARIA } & $4,4 \%$ & $7,5 \%$ & $3,1 \%$ \\
\hline \multicolumn{2}{|l|}{ 910: IX. Costes administrativos } & $5,7 \%$ & $4,0 \%$ & $-1,6 \%$ \\
\hline \multicolumn{2}{|l|}{ 920: X. Apoyo a las ONGs } & $0,5 \%$ & $0,3 \%$ & $-0,2 \%$ \\
\hline \multicolumn{2}{|l|}{ 930: XI. Refugiados } & $1,5 \%$ & $0,9 \%$ & $-0,6 \%$ \\
\hline \multicolumn{2}{|c|}{ 940: XII. Sin localizar ni especificar } & $24,6 \%$ & $6,2 \%$ & $-18,3 \%$ \\
\hline
\end{tabular}

Fuente: Elaboración propia con datos del CAD (2011b). Cálculos con desembolsos brutos de AOD en dólares constantes de 2009.

La cuota de participación del sector de "infraestructura y servicios económicos" creció 4,4 puntos porcentuales durante el periodo de Gobierno del PSOE, donde destaca el rubro de "transporte y almacenamiento" con un 5\%. Los "sectores productivos" descienden un punto porcentual por leves reducciones a las aportaciones en agricultura y minería.

Dentro del agregado "multisectorial" es donde se computan las contribuciones para el medio ambiente (410), que alcanzaron el 3\% en $2009 .{ }^{12}$ Las aportaciones del Gobierno socialista a la cooperación medioambiental multiplicaron por más de 2 la participación de estas ayudas en la AOD total, como consecuencia de la inclusión del medioambiente como prioridad horizontal del Plan Director 2005-2008.

12 El sector 430 "Otros multisectorial" es un agregado que incluye actuaciones de desarrollo urbano, desarrollo rural, desarrollo alternativo no agrario, enseñanza/formación multisectorial y financiación de instituciones científicas y de investigación. 
El peso de la ayuda para programas también creció durante el Gobierno del PSOE, siendo de interés el crecimiento del apoyo presupuestario, que pasó de un $0,01 \%$ en época del PP, a un $0,6 \%$ en época del PSOE. Asimismo, durante el Gobierno del PSOE se aumentó el peso de las operaciones de alivio de la deuda ( $+4,5$ puntos porcentuales en 2005-2009, hasta alcanzar el $13,7 \%$ de la AOD) y de ayuda humanitaria $(+3,1 \text { puntos porcentuales })^{13}$.

A pesar del incremento del volumen de fondos, en el periodo del PSOE se logró incurrir relativamente en menos costes administrativos $(-1,6 \%)$ y se financió en menor proporción a las ONGD $(-0,2 \%)$ y a los refugiados residentes en España $(-0,6 \%)$.

En resumen, la distribución sectorial ha sido relativamente estable en los últimos años, considerando los grandes niveles de agregación. En el periodo de Gobierno del PSOE han crecido de forma significativa las operaciones de alivio de la deuda externa, agua y saneamiento y gobierno y sociedad civil; y, de forma menos acentuada, la ayuda humanitaria y el apoyo presupuestario. En cambio, en época del PP los sectores productivos y la educación recibían mayores aportaciones relativas.

\subsection{Análisis de volatilidad de los sectores CAD-CRS en 5 dígitos}

El CAD clasifica las intervenciones de ayuda en 196 sectores desagregados a cinco dígitos. Utilizando esta información pormenorizada, el análisis se realiza en tres pasos: primero se computan el total de sectores en los que hubo intervención; en segundo lugar se estudian los principales estadísticos descriptivos para detectar cambios en el tiempo e identificar los sectores que han recibido más ayuda; y en tercer lugar se analiza la evolución de los 20 principales sectores de intervención.

Un primer dato relevante es la evolución del número de sectores en los que España aportó AOD (Figura 2). La evidencia es clara: a medida que ha aumentado el presupuesto de la AOD, se ha producido un aumento constante del número de sectores de intervención. Distinguiendo por periodos políticos, en promedio durante el Gobierno del PP (2002-2003) se desembolsó ayuda a 136,5 sectores por año, ascendiendo esta cifra a 161,4 en la etapa del PSOE (20052009). Este aumento puede ser producto de la creciente capacidad española de financiar intervenciones en un número mayor de sectores. Otra interpretación (o consecuencia) es que España no termina de especializarse sectorialmente y tiende a la dispersión, como resultado de una insuficiente planificación y eva-

\footnotetext{
${ }^{13}$ Un factor a tener en cuenta es el elevado peso del sector "sin especificar ni localizar" durante la etapa del PP (24,6\%), especialmente en 2002 (cuando alcanzó los 637 millones de dólares, el $32 \%$ de la AOD total). Es muy posible que este carácter residual se deba a cierta falta de estrategia sectorial que se ha ido subsanado en el tiempo, de tal modo que en el periodo del PSOE se redujo al 6,2\%la aportación de los sectores sin especificar.
} 
luación de las actuaciones que la permita discernir dónde tiene ventaja comparativa con respecto a otros donantes. Esta segunda interpretación parece acorde con la realidad de una ayuda orientada desde la oferta española de los fondos más que desde la demanda de los socios-receptores.

Figura 2

$N^{\circ}$ de sectores CAD en los que España aportó AOD

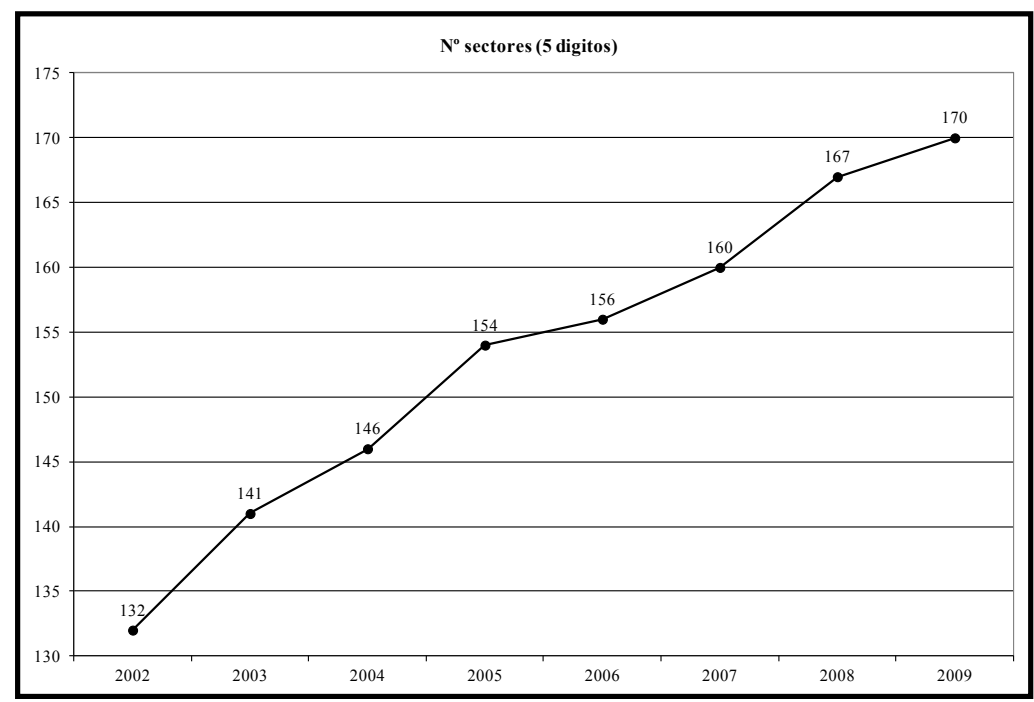

Fuente: Elaboración propia con datos del CAD (2011b). Cálculos con desembolsos brutos de AOD en dólares constantes de 2009. Se extraen las observaciones entre 2002-2009 (máximo disponible).

Asimismo, el análisis de los estadísticos descriptivos de la asignación sectorial de la AOD (promedios anuales de los desembolsos, desviación típica para observar la dispersión, y coeficiente de variación que orienta sobre la volatilidad en torno al promedio) para el periodo con datos sectoriales completos (20022009) ofrece información más matizada de esta tendencia a la dispersión sectorial (Figura 3). En primer lugar se observa -como era de esperar- que el promedio de desembolsos por sector incrementó (en términos contantes) desde los 15 millones de dólares de 2002 hasta los 28 millones en 2009. En segundo lugar, respecto a la dispersión de las asignaciones sectoriales, 2004 fue el año con menor desviación típica (26), mientras que en 2008 se alcanzó la mayor dispersión (superándose una desviación típica de 58). En tercer lugar, respecto a la volatilidad de las asignaciones, la evolución del coeficiente de variación permite establecer tres etapas. Desde 2002 a 2004 inclusive (bajo gestión del PP, salvo 2004 que fue año de comicios), donde la volatilidad fue minorándose, principalmente por el descenso del promedio de ayuda. Desde 2004 a 2006 (etapa de 
gestión del PSOE), cuando volvió a aumentar la volatilidad debido a que la desviación típica de las ayudas aumentó más rápido que el promedio de los desembolsos. Y una tercera etapa de 2007 a 2009 (de nuevo bajo gestión del PSOE), cuando la volatilidad se estabilizó en torno a $1,8 .^{14}$

Figura 3

Evolución de tres estadísticos descriptivos de la asignación sectorial de la AOD española

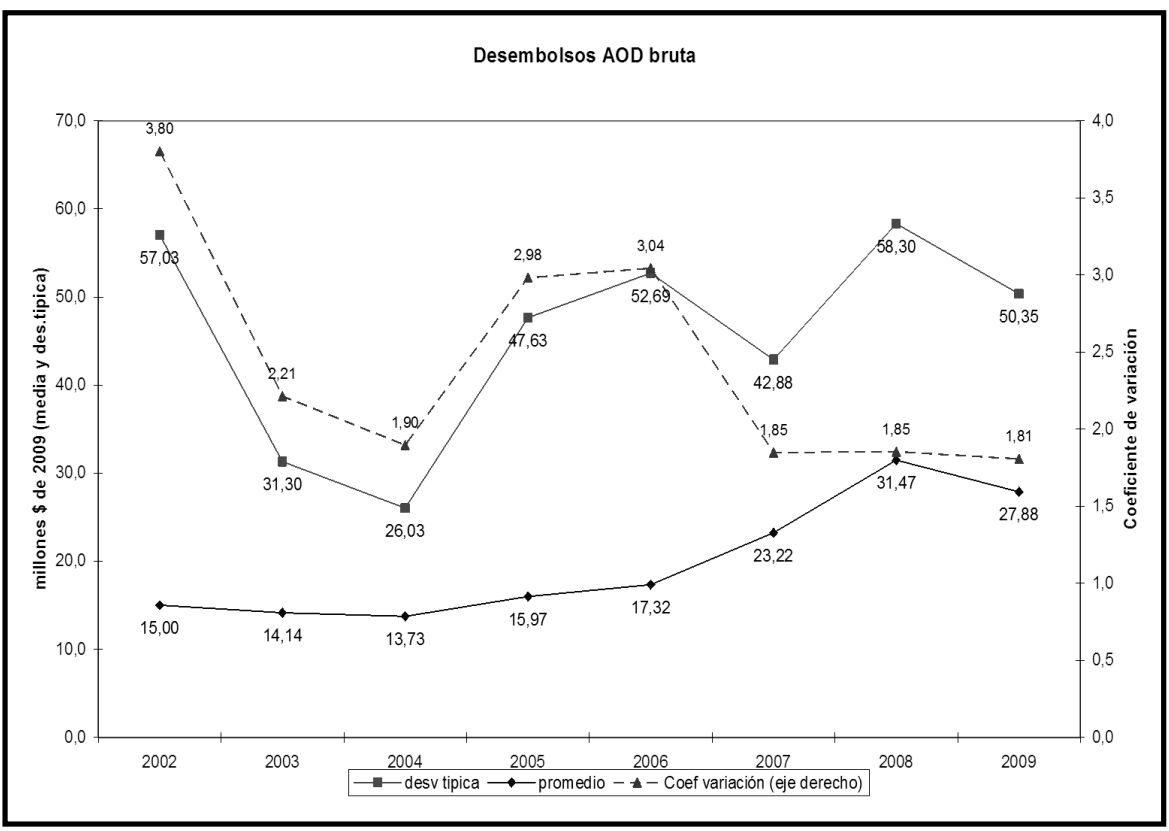

Fuente: Cálculos propios a partir de los datos del CAD-CRS. Dólares constantes de 2009.

\subsection{0 sectores con mayor financiación relativa}

Si no tenemos en cuenta los sectores definidos de forma residual (no especificados), ni los costes administrativos, podemos resaltar los siguientes hechos estilizados sobre los sectores con mayor promedio de ayuda entre 2002 y 2009 (Tabla 6):

${ }^{14}$ Por concepto, un coeficiente de variación de 1 significa que el incremento de desviación típica y promedio han sido idénticos. Un coeficiente de variación mayor que 1 implica que la desviación típica (dispersión entre los sectores) creció más que el promedio entre los sectores. 
Tabla 6

Ranking de los 20 sectores con mayor promedio de AOD 2002-2009

\begin{tabular}{|c|c|c|c|c|c|}
\hline Orden & Código y Sector & $\begin{array}{c}\text { Promedio } \\
\text { 2002-09 }\end{array}$ & $\begin{array}{c}\text { Promedio } \\
2002-03 \\
\text { PP }\end{array}$ & $\begin{array}{c}\text { Promedio } \\
2005-09 \\
\text { PSOE }\end{array}$ & $\begin{array}{c}\text { Dif. } \\
(05-09)-(02-03)\end{array}$ \\
\hline 1 & 60020: Condonación de deuda & $10,66 \%$ & $8,15 \%$ & $11,32 \%$ & $3,17 \%$ \\
\hline 2 & 99810: Sectores no especificados & $8,91 \%$ & $23,02 \%$ & $4,26 \%$ & $-18,77 \%$ \\
\hline 3 & 91010: Costes administrativos & $4,69 \%$ & $5,68 \%$ & $4,16 \%$ & $-1,52 \%$ \\
\hline 4 & 21030: Transporte por ferrocarril & $3,78 \%$ & $2,03 \%$ & $4,49 \%$ & $2,46 \%$ \\
\hline 5 & 72010: Ayuda de emergencia / Catástrofes & $2,78 \%$ & $1,85 \%$ & $3,15 \%$ & $1,30 \%$ \\
\hline 6 & $\begin{array}{l}\text { 14030: Abastecimiento básico de agua } \\
\text { potable y saneamiento }\end{array}$ & $2,49 \%$ & $1,47 \%$ & $3,23 \%$ & $1,77 \%$ \\
\hline 7 & 16061: Cultura y ocio & $2,37 \%$ & $2,34 \%$ & $2,34 \%$ & $0,00 \%$ \\
\hline 8 & 11420: Educación universitaria & $2,26 \%$ & $3,13 \%$ & $1,87 \%$ & $-1,26 \%$ \\
\hline 9 & $\begin{array}{l}\text { 15170: Organizaciones e instituciones de la } \\
\text { igualdad de las mujeres }\end{array}$ & $2,07 \%$ & $0,85 \%$ & $2,78 \%$ & $1,94 \%$ \\
\hline 10 & $\begin{array}{l}\text { 60061: Canje de deuda por proyectos } \\
\text { desarrollo }\end{array}$ & $2,06 \%$ & $0,45 \%$ & $2,87 \%$ & $2,41 \%$ \\
\hline 11 & $\begin{array}{l}\text { 15110: Política de administración y gestión } \\
\text { del sector público }\end{array}$ & $1,99 \%$ & $1,62 \%$ & $2,21 \%$ & $0,59 \%$ \\
\hline 12 & 73010: Reconstrucción y rehabilitación & $1,98 \%$ & $2,03 \%$ & $1,95 \%$ & $-0,08 \%$ \\
\hline 13 & 11220: Educación primaria & $1,97 \%$ & $1,52 \%$ & $2,33 \%$ & $0,82 \%$ \\
\hline 14 & $\begin{array}{l}\text { 16050: Multisectorial para servicios sociales } \\
\text { básicos }\end{array}$ & $1,79 \%$ & $2,12 \%$ & $1,65 \%$ & $-0,47 \%$ \\
\hline 15 & 43010: Ayuda Multisectorial & $1,74 \%$ & $1,10 \%$ & $2,15 \%$ & $1,05 \%$ \\
\hline 16 & $\begin{array}{l}\text { 99820: Fomento de sensibilización coop. al } \\
\text { desarrollo }\end{array}$ & $1,66 \%$ & $1,56 \%$ & $1,74 \%$ & $0,18 \%$ \\
\hline 17 & 43040: Desarrollo Rural & $1,56 \%$ & $1,57 \%$ & $1,42 \%$ & $-0,16 \%$ \\
\hline 18 & $\begin{array}{l}\text { 52010: Ayuda alimentaria / Seguridad } \\
\text { alimentaria }\end{array}$ & $1,52 \%$ & $0,73 \%$ & $1,98 \%$ & $1,25 \%$ \\
\hline 19 & 11330: Formación profesional & $1,50 \%$ & $2,06 \%$ & $1,30 \%$ & $-0,76 \%$ \\
\hline 20 & 16010: Seguridad Social/Servicios sociales & $1,28 \%$ & $1,19 \%$ & $1,34 \%$ & $0,15 \%$ \\
\hline
\end{tabular}

Fuente: Elaboración propia con datos del CAD (2011). Porcentajes calculados respecto del total de ayuda desembolsada sectorialmente. Sectores ordenados por el promedio 2002-2009.

- Mayor protagonismo de las "operaciones de deuda" (condonación y canje), durante el Gobierno del PSOE que en la etapa del PP. Debe tenerse en cuenta que varias de éstas operaciones son resultado de decisiones internacionales y no tanto de opciones discrecionales de partido político.

- El sector de "transporte por ferrocarril" (nunca detectado anteriormente como prioritario) ocupa el cuarto lugar. Se produce un incremento notable entre los años 2005 y 2009 (durante la etapa socialista), donde llega a representar el 4,5\% de la AOD. El país protagonista de estos desembolsos fue Turquía, que recibió en promedio (para todo el periodo) el 52\% de la AOD de este sector (988 millones de dólares). Además Turquía representa más de la mitad del destino del transporte por ferrocarril todos los años (la 
única excepción es 2005). A Turquía le siguen Argelia (21\%), China (18\%), Egipto (3\%), Bosnia-Herzegovina (3\%), Kazajstán (1\%), Marrue$\cos (0,3 \%)$, Túnez $(0,1 \%)$ y una pequeña dotación a Etiopía en 2009 (apenas 10.000 dólares). Por cierto, ni Turquía ni China fueron priorizados el Plan Director 2009-2012, y a ellos fue a parar el 77\% de la AOD al sector de transporte por ferrocarril en 2009.

- Importante peso de la "acción humanitaria" (el agregado de las ayudas de emergencia, reconstrucción y alimentarias), que en el ranking del promedio 2002-09 ocuparon los puestos quinto, decimosegundo y decimoctavo. En concreto, la ayuda de emergencia experimentó un incremento notable $(1,3 \%)$ en el periodo del PSOE, hasta alcanzar el 3\% de la AOD. La alimentaria creció 1,25 puntos porcentuales en este mismo periodo (200509), lo que evidencia la apuesta política y estratégica por la especialización en este sector, directamente relacionado con el primer Objetivo de Desarrollo del Milenio (reducir a la mitad el número de hambrientos). El peso político otorgado por el PSOE a las Reuniones de Alto Nivel sobre Seguridad Alimentaria refuerzan esta apuesta, por lo que sería positivo que se mantuviera y se profundizaran las evaluaciones que detecten las causas y mejores instrumentos que combaten la inseguridad alimentaria. En estas evaluaciones se debería valorar si las intervenciones en desarrollo rural (decimoséptimo en el ranking de los 20) ejercen externalidades positivas consistentes y complementarias a la ayuda alimentaria.

- Los incrementos de 2008 y 2009 en la dotación al Fondo Español para Agua y Saneamiento, con 360 y 247 millones de dólares respectivamente, explican el paso de un promedio de $1,47 \%$ en el periodo de gestión del PP, al $3,23 \%$ en el periodo del PSOE.

- En séptima posición se sitúa el sector "cultura y ocio", con la misma participación promedio del 2,34\% tanto en el periodo del PP como del PSOE, si bien debe notarse la diferencia en términos absolutos, con 47 millones de dólares de promedio durante el Gobierno del PP y 97 millones (más del doble) de promedio en época del PSOE. Destaca la volatilidad en los años de gestión del PSOE, pues mientras en 2006 lo aportado fueron 54 millones -en línea con los anteriores ejercicios-, los desembolsos de 2007 y 2008 fueron de 165 y 107 millones de dólares, respectivamente. Pero en 2009 cayó a menos de 62 millones de dólares. El dato puede confirmar un modelo de ayuda dirigido por la oferta española y con fuerte peso de los programas de conservación de patrimonio, escuelas taller o promoción del idioma español. Esta situación en el ranking de prioridades sectoriales bien merecería una evaluación temática en profundidad.

- En los puestos octavo y decimotercero aparecen sectores educativos. Este protagonismo está en línea con las directrices de los Planes Directores y el 
lugar común de la educación como base del desarrollo de largo plazo. Lo que se puede valorar de forma más crítica es que los fondos de educación superior casi duplicaran a los de educación primaria en los ejercicios de gestión del PP de 2002 a $2004^{15}$. Además las aportaciones a universitarios se han mantenido constantes en todos los años en torno al promedio de los 62 millones de dólares. La gestión del PSOE durante el Plan Director 2005-08 apostó de forma más decidida por fortalecer la educación primaria -en línea con los Objetivos de Desarrollo del Milenio 2 y 3-, con un incremento de 0,82 puntos porcentuales frente a la del PP; en cambio, se redujo la aportación a los programas de becas y de apoyo a la educación superior, que experimentaron un descenso de $-1,26$ puntos porcentuales. Finalmente, la formación profesional ocupa el puesto decimonoveno en el ranking de 2002-2009, descendiendo 0,76 puntos porcentuales durante la gestión del PSOE. El hecho de que existan tres subsectores dedicados a la educación entre los 20 primeros es un dato a valorar positivamente, pero debería avanzarse en la especialización dentro del amplio sector educativo y fomentar las evaluaciones de impacto (qué funciona y qué no en la mejora del aprendizaje, calidad y utilidad de la educación para el empleo en los países socios, por ejemplo).

- El noveno puesto lo ocupa el "apoyo a organizaciones que promueven la igualdad de la mujer". Éste es otro de los sectores enfatizados por la administración del PSOE. Durante el Gobierno del PP el promedio de participación en los fondos fue del $0,85 \%$, mientras que en el periodo del PSOE ascendió al 2,78\%. De nuevo podemos identificar una apuesta sectorial estratégica por parte del PSOE que no se había hecho anteriormente.

- Análogamente, durante el Gobierno del PSOE se apoyó de manera diferencial el subsector de la "política de administración y gestión del sector público" (puesto decimoprimero), siendo uno de los que incluye a una de las principales causas de la pobreza: la debilidad institucional y el mal gobierno (Larrú, 2006 y 2009). También fue diferencial la aportación a la "sensibilización y educación para el desarrollo", pasando de un $1,56 \%$ a un $1,74 \%$ de la AOD española.

- Un último apunte: el primer sector relacionado con la salud se sitúa en el puesto 26 (salud básica), con un porcentaje del 1,15\%.

En resumen, la ayuda española tiene una alta dispersión entre el elevado número de sectores en los que el CAD clasifica los flujos. El Gobierno del PSOE consiguió una menor volatilidad sectorial en la distribución del conjunto de la

\footnotetext{
${ }^{15}$ Mientras la educación superior recibía montos superiores a los 60 millones de dólares, la primaria apenas superó los 30. El mayor desequilibrio se produce en 2004, con 20 millones a primaria frente a 50 a superior.
} 
AOD española (con un coeficiente de variación de 2,3, frente al 3 alcanzado durante el Gobierno del PP). Esta elevada variabilidad en las asignaciones sectoriales ha permitido a España intervenir ante múltiples necesidades, pero a costa de una insuficiente especialización y actuación bajo ventajas comparativas. El proceso comunitario de especialización y división del trabajo entre los donantes europeos conduce a la especialización ${ }^{16}$, con lo que España deberá concentrar sus intervenciones en aquellos sectores en los que tenga ventajas comparativas, o en aquellos en los que -bajo discrecionalidad política- decida hacerlo de forma estratégica.

Del análisis de los 20 sectores que han recibido -en promedio- mayor volumen de AOD se desprenden tres hechos estilizados: i) una fuerte presencia de operaciones de deuda; ii) el protagonismo de ocho sectores específicos (ferrocarril; mujer; agua; educación; cultura y ocio; acciones humanitarias; seguridad alimentaria y desarrollo rural; y fortalecimiento del sector público); y iii) una mayor concentración de fondos en estos ocho sectores específicos durante la etapa del PSOE. La Figura 4 expresa esos incrementos diferenciales respecto de la etapa del PP.

\section{INSTRUMENTOS DE LA AOD ESPAÑOLA}

Por último, cabe apuntar brevemente cuál es la distribución de la AOD española en función de los diferentes tipos y modalidades de ayuda que identifica la base de datos del CAD. Al disponerse de series temporales más largas, podemos analizar los periodos de gestión 1997-2003 (PP) y 2005-2009 (PSOE).

El grueso de la AOD española en ambas etapas de gestión ha consistido en "ayuda no reembolsable" (es decir, donaciones) (Tabla 7). No obstante, las donaciones experimentaron un importante incremento en la etapa socialista, pasando de representar el $79 \%$ al $98 \%$ de los desembolsos netos de AOD, principalmente por la ampliación de las ayudas alimentarias, de emergencia y la cooperación técnica. Entre las donaciones, la cooperación técnica se ha consagrado como la principal modalidad no reembolsable, especialmente durante la gestión del PSOE, cuando se alcanzó una cuota promedio del 20,4\% de la AOD neta. En menor medida destacan las operaciones de alivio de la deuda, que fue-

\footnotetext{
${ }^{16}$ De hecho, el Código de Conducta de la Unión Europea relativo a la división del trabajo en el ámbito de la política de desarrollo (Comisión Europea, 2007) identifica cuatro principios específicos para avanzar en la especialización de los donantes: $i$ ) concentrar las actividades en el país en dos sectores prioritarios; ii) reorganizar las demás actividades en el país (cooperación delegada, apoyo presupuestario o retirada responsable); iii) velar por una presencia adecuada de la UE en sectores estratégicos (limitando para 2010 el número de donantes activos a un máximo de tres a cinco por sector dentro de cada país en cuestión); y iv) establecer países prioritarios focalizando la ayuda, con un esfuerzo suplementario en favor de los países huérfanos que frecuentemente son estados frágiles infra-financiados.
} 
ron ligeramente mayores durante el periodo del PP, en parte como consecuencia de la macro-operación de condonación de la deuda de Nicaragua en 2001.

Figura 4

Evolución de la AOD a los principales sectores CAD-CRS: diferencias porcentuales entre las asignaciones durante las etapas de Gobierno de PP y PSOE

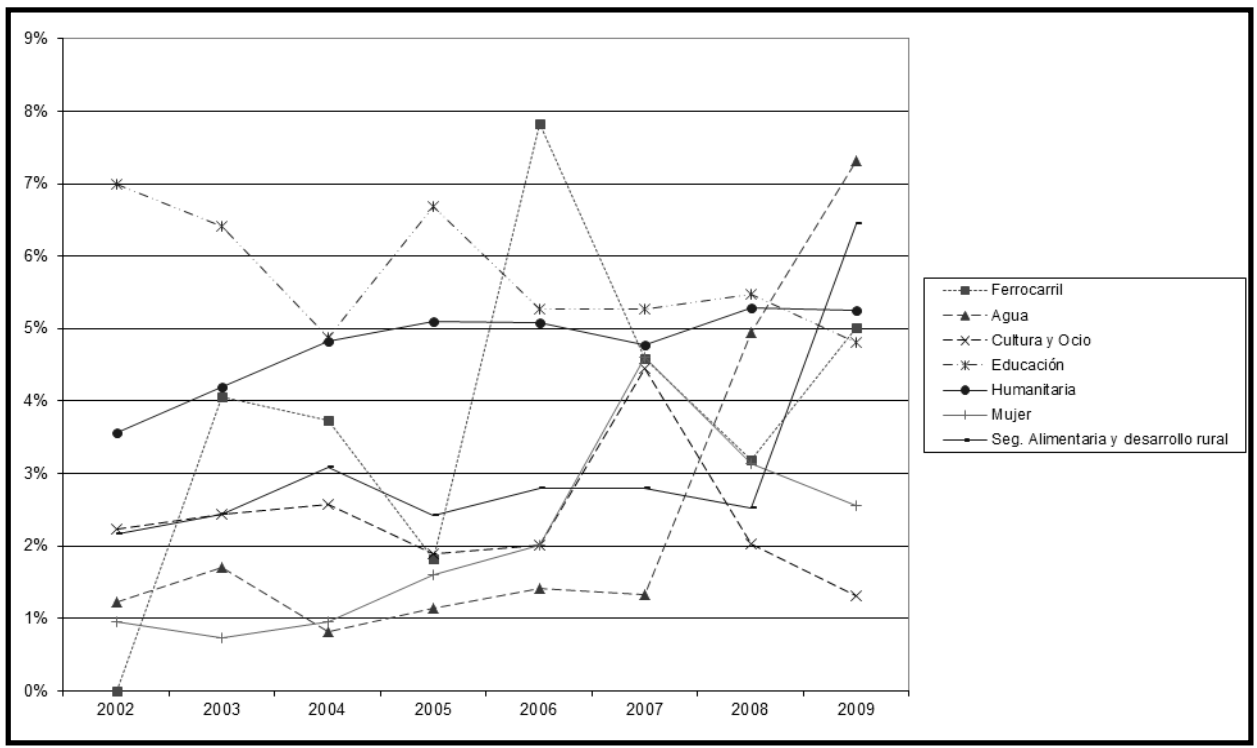

Fuente: Elaboración propia con datos del CAD (2011) sobre los desembolsos brutos en dólares constantes de 2009. Educación incluye la suma de educación superior, primaria y formación profesional. Ayuda humanitaria incluye emergencia y reconstrucción. Se excluye deuda externa.

Como consecuencia del creciente énfasis en las donaciones, se ha reducido la participación de la "ayuda reembolsable" (préstamos), que, en términos netos, supuso en el periodo del PSOE poco más del $2 \%$ de las ayudas (frente al $20 \%$ del periodo del PP). Esta menor utilización del crédito como instrumento de cooperación en la etapa socialista se debe tanto a una reducción de los desembolsos de nuevos créditos (menos de la mitad, en términos relativos, que en la etapa del PP), como a una importante minoración del pasivo que genera la ayuda reembolsable (reembolso de préstamos anteriores y pago de intereses).

Esta tendencia parece reflejar la apuesta del Gobierno socialista por las donaciones (en detrimento de los créditos concesionales), tal y como han reclamado diversos sectores de la cooperación española. No obstante -cabe alertar-, España no dispone de evaluaciones que la permitan identificar en qué países y contextos socio-económicos son preferibles las ayudas reembolsables o las no reembolsables. Se trata, pues, de un ámbito en el que España habrá de dotarse 
de evidencia empírica para poder diseñar una estrategia instrumental clara; estrategia que deberá contemplar la peculiar especialización de la cooperación española en los países de renta media, entre los que puede ser oportuno (en determinadas intervenciones, especialmente aquellas orientadas a financiar grandes infraestructuras) el uso de créditos concesionales. ${ }^{17}$

Tabla 7

Instrumentos y modalidades de AOD española. 1997-2003 y 2005-09 (\%)

\begin{tabular}{|c|c|c|c|}
\hline & \multicolumn{2}{|c|}{$\operatorname{AOD}(\%)^{(1)}$} & \multirow{3}{*}{ Variación (\%) } \\
\hline & $\begin{array}{c}1997-2003 \\
\text { PP }\end{array}$ & $\begin{array}{l}2005-2009 \\
\text { PSOE }\end{array}$ & \\
\hline TOTAL AOD neta & 100 & 100 & \\
\hline $\begin{array}{l}\text { Total donaciones } \\
\text { Entre otras: } \\
\text { Condonaciones deuda } \\
\text { Ayuda alimentaria } \\
\text { Ayuda humanitaria } \\
\text { Cooperación técnica }\end{array}$ & $\begin{array}{r}79.67 \\
15.45 \\
0.63 \\
3.62 \\
16.95\end{array}$ & $\begin{array}{r}97.79 \\
14.11 \\
2.46 \\
8.20 \\
20.39 \\
\end{array}$ & $\begin{array}{r}18.11 \\
-1.33 \\
1.82 \\
4.58 \\
3.44 \\
\end{array}$ \\
\hline $\begin{array}{l}\text { Total prestamos netos } \\
\text { Entre otros: } \\
\text { Prestamos brutos } \\
\text { Reembolsos de prestamos } \\
\text { Renegociación de deuda } \\
\text { Ajustes contables por operaciones alivio deuda }\end{array}$ & $\begin{array}{r}20.33 \\
37.53 \\
-14.74 \\
0.52 \\
-2.47\end{array}$ & $\begin{array}{r}2.21 \\
15.64 \\
-8.15 \\
0.21 \\
-5.27\end{array}$ & $\begin{array}{r}-18.11 \\
-21.90 \\
6.59 \\
-0.31 \\
-2.80\end{array}$ \\
\hline $\begin{array}{l}\text { Memo: } \\
\text { Cobro de intereses } \\
\text { AOD total, excl. deuda } \\
\text { Alivio neto deuda }\end{array}$ & $\begin{array}{l}-3.96 \\
86.50 \\
13.50\end{array}$ & $\begin{array}{l}-1.33 \\
89.14 \\
10.86\end{array}$ & $\begin{array}{r}2.63 \\
2.63 \\
-2.63\end{array}$ \\
\hline
\end{tabular}

(1) Cuota por instrumento en el desembolso neto acumulado de AOD por periodo (dólares constantes 2009).

Fuente: Elaboración propia con datos del CAD (2011b).

\section{CONCLUSIONES}

España cierra un ciclo de fuerte expansión de su política pública de cooperación internacional para el desarrollo, e inicia una nueva etapa de crisis y ajuste presupuestario. La crisis de financiación de la AOD ofrece la "oportunidad" de iniciar un proceso de "destrucción creativa" -en el sentido schumpeteriano- que permita revisar los aspectos "económicos" de la distribución de los recursos tanto a nivel geográfico, como a nivel sectorial e instrumental- con el objetivo

\footnotetext{
${ }^{17}$ Véase, a este respecto, el estudio de eficacia de la ayuda desarrollado por Tezanos et al. (2012), en el que se estima que los impactos ejercidos por las donaciones y por los créditos concesionales han sido positivos para el ritmo de crecimiento de los países latinoamericanos y caribeños, si bien los créditos han ejercido estímulos proporcionalmente mayores que las donaciones.
} 
de definir una "estrategia de especialización" de la cooperación pública española que sea verdaderamente selectiva y eficaz. Se trata, por tanto, del momento adecuado para afrontar, de manera decidida, el reto de actualizar la estrategia española de cooperación para tratar de compensar -al menos parcialmente- la importante reducción de los recursos con aumentos en el impacto y la calidad de las actuaciones -calidad, por otro lado, frecuentemente cuestionadas por los indicadores internacionales-.

Este artículo estudia por ello la distribución geográfica, sectorial e instrumental de la AOD española, con el propósito de esclarecer cuáles son las causas de su excesiva dispersión e identificar en qué países, sectores e instrumentos se "especializa" de facto.

Dada la pluralidad de actores públicos que financian la AOD española (AGE, CCAA, EELL y universidades), este artículo analiza el nivel de coordinación logrado por los sucesivos Gobiernos de España -del PP y del PSOE- en términos de la distribución (geográfica, sectorial e instrumental) del conjunto de la AOD española, con el propósito de esclarecer cuáles son las causas de su excesiva dispersión e identificar en qué países, sectores e instrumentos se "especializa" de facto. Para ello, el análisis parte de la constatación de que la AGE (y en concreto el Ministerio de Asuntos Exteriores y Cooperación) es responsable de la coordinación de la política española de cooperación, tal y como define la Ley Española de Cooperación Internacional para el Desarrollo.

De forma global, durante el Gobierno del PP se gestionó una política pública de cooperación bastante inercial (respecto a lo que se venía haciendo), asumiendo como "seguidor" las directrices comunitarias. El Gobierno del PSOE, sin embargo, consideró la cooperación para el desarrollo como uno de los principales componentes de la política exterior, concediéndola mayor protagonismo político y aumentando considerablemente los recursos. No obstante, durante el Gobierno socialista no se logró incrementar la calidad y el conocimiento sobre el impacto de las ayudas (en términos de desarrollo en los países socios), ni se lograron identificar las ventajas comparativas españolas respecto del resto de donantes -sobre todo comunitarios-, lo que limitó las posibilidades de mejora en los rankings internacionales de calidad de los donantes.

Los principales resultados de la investigación se resumen a continuación:

En relación con la distribución geográfica, el mapa de la AOD española se ha caracterizado por su inclinación histórica hacia los países en desarrollo de América (región históricamente vinculada a España) y del norte de África (por su proximidad geográfica). Estos vínculos históricos y políticos de España explican la inclinación hacia los países de renta media, que siguen siendo (especialmente los países de renta media-baja) los principales receptores. No obstante, la principal diferencia entre los periodos de gestión del PP y del PSOE ha sido la apuesta del Gobierno socialista por incrementar las ayudas a los paí- 
ses más pobres, incorporando a nuevos socios del África subsahariana (en línea con las prioridades de la agenda de los Objetivos de Desarrollo del Milenio).

No obstante -cabe alertar- el número de países socios de la cooperación oficial española no ha cesado de aumentar con el tiempo (coincidiendo con la expansión de los recursos gestionada por el Gobierno del PSOE), lo que agrava los problemas de fragmentación y volatilidad de la AOD y merman el impacto global de las ayudas. Por eso, es necesaria una concentración de los recursos en torno a un número reducido de países socios realmente prioritarios, que deberían identificarse de manera transparente (combinando criterios políticos, técnicos y de desarrollo). La evidencia revela la existencia de una concentración de facto en torno a una docena de países, a pesar de que los dos últimos Planes Directores priorizaron a más de 50. Así, con una lista corta de entre 10 y 12 países verdaderamente prioritarios se gestionaría, aproximadamente, la mitad del presupuesto de AOD.

Respecto a la distribución sectorial, el reparto ha sido relativamente estable en los últimos años, si bien en el periodo de Gobierno del PSOE crecieron de forma significativa las aportaciones a los sectores de alivio de la deuda externa, agua y saneamiento, gobierno y sociedad civil, ayuda humanitaria y apoyo presupuestario. En cambio, en época del PP los sectores productivos y la educación (especialmente la universitaria) recibían mayores aportaciones relativas. Asimismo, el Gobierno del PSOE logró mejorar la concentración sectorial de los fondos y redujo la volatilidad de las asignaciones. No obstante, el Gobierno socialista no supo evitar que la ampliación del presupuesto de AOD se tradujera en un aumento permanente del número de sectores de intervención. Este aumento refleja una estrategia inacabada de especialización sectorial, que tiende a la dispersión, como resultado de la insuficiente planificación y evaluación de las actuaciones, a pesar de que estas actividades son necesarias para identificar las ventajas comparativas sectoriales de España respecto a otros donantes internacionales. Como resultado, la asignación sectorial ha estado históricamente orientada desde la oferta española de los fondos (interesada en intervenir en múltiples sectores) más que desde la demanda de los socios.

Para resolver esta carencia estratégica, España deberá concentrar sus intervenciones en un número reducido de sectores. En este ámbito, el Código de Conducta de la UE relativo a la división del trabajo en el ámbito de la política de desarrollo recomienda concentrar las actividades de cada donante en dos sectores prioritarios (dentro de cada país socio) y velar por una presencia adecuada de la UE en los "sectores estratégicos" (con un máximo de entre tres y cinco donantes activos por sector dentro de cada país socio). En esta línea, la evidencia revela la existencia de nueve sectores donde España ha tendido a concentrar su ayuda: alivio de la deuda; ferrocarril; mujer; agua; educación; cultura 
y ocio; acciones humanitarias; seguridad alimentaria y desarrollo rural; y fortalecimiento del sector público.

Por último, en relación con los instrumentos y modalidades de cooperación, el grueso de la AOD española consiste en "ayudas no reembolsables" (donaciones), y esta modalidad ha tendido a reforzarse en la etapa de gestión del PSOE (principalmente por la ampliación de las ayudas alimentarias, de emergencia y la cooperación técnica), siendo la cooperación técnica la principal modalidad no reembolsable. Consiguientemente, las "ayudas reembolsables" (préstamos) han perdido peso en la AOD española, debido a la reducción de los desembolsos de nuevos créditos y a la minoración del pasivo de la ayuda reembolsable (reembolso de préstamos y pago de intereses). No obstante, la apuesta del Gobierno socialista por las donaciones (en detrimento de los créditos concesionales, que tuvieron un peso elevado durante la gestión del PP) debe acompañarse de la generación de evidencia empírica que permita diseñar una estrategia instrumental clara que identifique en qué países y contextos socio-económicos son preferibles las ayudas reembolsables o las no reembolsables, partiendo de la especificidad española de que la mayor parte de sus socios son países de renta media entre los que puede ser oportuno (en determinadas circunstancia) el uso de créditos concesionales.

\section{REFERENCIAS BIBLIOGRÁFICAS}

COMISIÓN EUROPEA (2007): Código de Conducta de la Unión Europea relativo a la división del trabajo en el ámbito de la política de desarrollo, COM (2007) 72 final, Comisión de las Comunidades Europeas, Bruselas.

COMITÉ DE AYUDA AL DESARROLLO (CAD) (2002), Development co-operation review 2002: Spain, OECD, Development Assistance Committee, París.

COMITÉ DE AYUDA AL DESARROLLO (CAD) (2007), Development co-operation review 2007: Spain, OECD, Development Assistance Committee, París.

COMITÉ DE AYUDA AL DESARROLLO (CAD) (2011a), Development cooperation review 2011: Spain, OECD, Development Assistance Committee, París.

COMITÉ DE AYUDA AL DESARROLLO (CAD) (2011b), Development Database on Aid from DAC Members: DAC online, OECD.Stat, disponible en http://www.oecd.org/dataoecd/

DARA (2011) Humanitarian Response Index 2011. Addressing the gender challenge. DARA. Madrid. 
DJANKOV, S., MONTALVO, J.G. y REYNAL-QUEROL, M. (2009): “Aid with Multiple Personalities" en Journal of Comparative Economics, 37, pp. 217229.

DURAN, D. y GLASSMAN, A. (2012): "An Index of the Quality of Official Development Assistance in Health" en Center for Global Development Working Paper, 287.

LARRÚ, J.M. (2006): "Ayuda al Desarrollo y buen gobierno en los países de Renta Media. Recomendaciones para España" en Revista Electrónica Instituciones y Desarrollo, 17 (1), pp. 1-18.

LARRÚ, J.M. (2009): "Corrupción y Ayuda al Desarrollo. Evidencias, Teoría y Aplicaciones para España” en Cuadernos Económicos de ICE, 78, pp. 261280.

LARRÚ, J.M. (2011): “¿Más es mejor? Reflexiones en torno a la calidad de la ayuda al desarrollo española" en Revista de Economía Mundial, 28, pp. 205246.

MINISTERIO DE ASUNTOS EXTERIORES (MAE) (2001): Plan Director de la Cooperación Española 2001-2004, disponible en http://www.aecid.es/es/ servicios/publicaciones

MINISTERIO DE ASUNTOS EXTERIORES (MAE) (2005): Plan Director de la Cooperación Española 2005-2008, disponible en http://www.aecid.es/es/ servicios/publicaciones

MINISTERIO DE ASUNTOS EXTERIORES Y COOPERACIÓN (MAEC) (2009): Plan Director de la Cooperación Española 2009-2012, disponible en http://www.aecid.es/es/servicios/publicaciones

OLIVIÉ, I., CASCANTE, K., DOMÍNGUEZ, R., LARRÚ, JM., SOTA, J. y TEZANOS, S. (2011): "Nunca desaproveches una buena crisis: hacia una política pública española de desarrollo internacional" en Informes Elcano, 13, Real Instituto Elcano, Madrid

TEZANOS, S. (2008a): Cooperación para el desarrollo. Asignación geográfica de la ayuda española, Madrid: Biblioteca Nueva.

TEZANOS, S. (2008b): "Aiding middle-income countries? The case of Spain" en Oxford Development Studies, 36(4), pp. 409-438.

TEZANOS, S. y GUTIÉRREZ, D. (2011): "Altruismo o interés? El papel de la inmigración en la asignación geográfica de la ayuda oficial española al desarrollo" en Documentos de trabajo sobre cooperación y desarrollo, 2011/03, Cátedra de Cooperación Internacional y con Iberoamérica, Universidad de Cantabria, disponible en http://www.ciberoamericana.unican.es/ workingpapers.htm

TEZANOS, S., GUIJARRO, M. y QUIÑONES, A. (2012): “Inequality, aid and growth: macroeconomic impact of aid grants and loans in Latin America and the Caribbean" en Journal of Applied Economics (en prensa). 
TEZANOS, S., MADRUEÑO, R. y GUIJARRO, M. (2009): "Impacto de la ayuda sobre el crecimiento económico. El caso de América Latina y el Caribe" en Cuadernos Económicos, Información Comercial Española, 78, pp. 187-220.

TORRES, T. y ALLEPUZ, R. (2009): "El desarrollo humano: perfiles y perspectivas futuras" en Estudios de Economía Aplicada, 27-2, pp. 545-562. 